ARTICLE

\title{
Application of the Tikhonov Unfolding Method for Reconstruction of Primary X-Ray Spectra from X-Ray Equipments
}

\author{
Andrea QUEROL ${ }^{*}$, Sergio GALLARDO, José RÓDENAS and Gumersindo VERDÚ \\ Departamento de Ingeniería Química y Nuclear, Universidad Politécnica de Valencia, \\ Camino de Vera s/n 46022 Valencia, Spain
}

\begin{abstract}
Quality Control (QC) parameters for an X-ray tube depend strongly on the accurate assessment of the primary spectrum. In previous works, a methodology to assess primary spectrum using a Compton spectrometry technique has been analyzed. This methodology consists in the use of a Monte Carlo (MC) model, by means of the MCNP5 code, to reproduce the physical phenomena involving the interaction of photons and electrons with the Compton spectrometer and a High-Purity Germanium detector (HPGe). The relation between the Pulse Height Distribution (PHD) recorded by the detector and the primary beam X-ray spectrum is defined by a Response matrix. To estimate the primary spectrum knowing the PHD is necessary to obtain the inverse of the Response matrix. This is an inefficient task using traditional methods because this matrix is ill-conditioned. In this paper, the Tikhonov method has been used to obtain an approximation to this matrix. This unfolding mathematical tool requires a sensitivity study to know the effect of the truncation process. This methodology can be very useful for QC of X-ray tubes used in radiodiagnostic.
\end{abstract}

KEYWORDS: X-ray spectra, quality control, Compton spectrometry, Tikhonov unfolding method

\section{Introduction}

It is necessary an accurate assessment of the primary spectrum for a good QC of X-ray tubes, but obtaining primary beam spectrum experimentally implies some important difficulties. The use of direct spectrometry for determining primary X-ray spectrum is practically forbidden as detectors cease to work properly at high count rates. To avoid the pile-up effect in the detector produced by a high fluence rate, a Compton spectrometry technique was proposed by Matscheko and Ribberfors. ${ }^{1)}$ In a previous work, ${ }^{2)}$ authors described a Monte Carlo model using the MCNP $\operatorname{code}^{3)}$ to simulate the spectrometry process by obtaining the Pulse Height Distribution (PHD) for different tube working conditions.

The spectrum obtained with the spectrometry technique needs to be unfolded since some effects such as photon interactions, efficiency variations or perturbations from electronic devices, produce a distortion in the actual spectrum. The response function of the process is approximated by a Response matrix containing all the required information to unfold the PHD measured.

It is however an ill-conditioned matrix and requires some special mathematical treatment. In a previous work, ${ }^{4)}$ authors described the Modified Truncated Singular Value Decomposition (MTSVD) unfolding method and its application to the Compton scattering.

In this work, the Tikhonov method has been applied to unfold spectra in the radiodiagnostic energy range. The main goal is to test the method goodness by comparing obtained unfolded spectra with actual primary ones. The method has

*Corresponding author, E-mail: anquevi@upvnet.upv.es been applied for different X-ray tube working conditions and a sensitivity analysis has been performed by varying high voltage, anode angle and filter thickness.

Differences have been quantified by the Root Mean Squared (RMS) and also by obtaining the first and second Half Value Layer (HVL) in air kerma, the homogeneity factor and the mean energy of each spectrum.

\section{Methodology}

The methodology used can be summarized into four main steps, as it can be seen in Fig. 1.

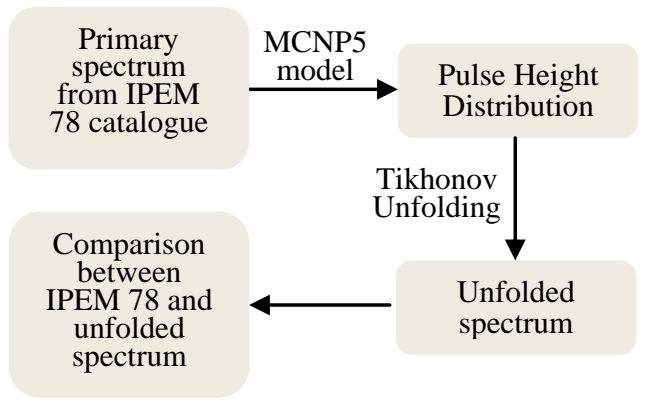

Fig. 1 Schematics of the methodology used

First, IPEM78 Catalogue C) $^{5}$ has been used to define a theoretical primary spectrum. Different cases have been considered varying the above mentioned three working conditions for the X-ray tube. Secondly, this information has been used to define the X-ray source in the MCNP5 model developed. After running the code, a simulation of the PHD recorded in the detector is obtained. Thirdly, Tikhonov me-

(C) 2011 Atomic Energy Society of Japan, All Rights Reserved. 


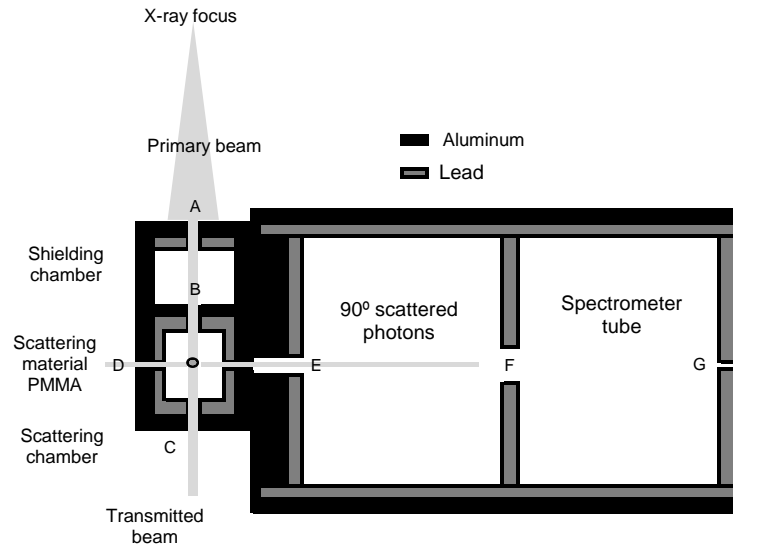

Fig. 2 The Compton spectrometer layout

thod has been applied to the PHD, obtaining an unfolded spectrum. Finally, a comparison between original IPEM 78 and unfolded spectrum is done.

\section{The Monte Carlo Model}

The MCNP5 model includes the X-ray focus, a Compton spectrometer and a high purity Germanium (HPGe) detector. The geometry of the MCNP5 model is shown in Fig. 2 where the PMMA scattering rod, chambers and collimators can be seen. The dimensions of the collimators are $4 \times$ $40 \mathrm{~mm}$ at entrance sides and towards the detector and $5 \times$ $45 \mathrm{~mm}$ at exit sides. ${ }^{6)}$

MCNP5 is suitable for modeling the detector response, since it contains a tally, F8, which is specifically designed for detector pulse height determination. Detector resolution has been taken into account in the simulation by choosing an adequate number of energy bins. In addition, a Gaussian Energy Broadening (GEB) function of MCNP has been used to reproduce the real detector FWHM (full width at half maximum).

Coherent scattering has been disabled because its effects are minimized using a scattering rod of low- $Z$ and a $90^{\circ}$ scattering angle. Electrons have been taken into account with the TTB (Thick Target Bremsstrahlung) option in MCNP5. This option helps to reduce computing time. This approach is acceptable due to the small mean free path in air of electrons produced in shielding. A photon cut off has been set to $1 \mathrm{keV}$ (default value).

The Germanium detector simulated is a Canberra Ultra Low Energy Germanium detector ${ }^{7)}$ with high detection efficiency in the range corresponding to radiodiagnostic energies. The beryllium window is $0.05 \mathrm{~mm}$ thick. The main features of this detector are: $50 \mathrm{~mm}^{2}$ of active area and $5 \mathrm{~mm}$ of thickness. The resolution is $145 \mathrm{eV}$ (FWHM) for $5.9 \mathrm{keV}$ (Fe-55) and $500 \mathrm{eV}$ (FWHM) for $122 \mathrm{keV}$ (Co-57).

The efficiency of the detector presents a wide plateau between 20 and $100 \mathrm{keV}$. The K-edge at $11 \mathrm{keV}$ produces a local decrease in efficiency.

\section{The Unfolding Method}

The relation between the PHD and the primary spectrum is defined by a Response function, usually expressed as a matrix. That is,

$$
R \cdot \vec{s}=\vec{m}
$$

where

$s \quad$ is the unknown primary spectrum,

$m$ is the PHD recorded in the detector system and

$R$ is the Response matrix.

$R$ can be obtained with $1 \mathrm{keV}$ resolution from PHD's calculated by simulating several monochromatic photon beams with MCNP.

Once the Response matrix is known, Eq. (1) theoretically permits to obtain the primary spectrum $\vec{s}$. However, $R$ is ill-conditioned (condition number $9.310^{20}$ ) and this implies that it is very difficult to obtain $R^{-1}$ with a standard numerical procedure. An approximation to $R^{-1}$ should be obtained and the Tikhonov method has been chosen ${ }^{8)}$ for this purpose.

Tikhonov regularization method ${ }^{9-11)}$ is based on a modification of the Fredholm integral equation of first order that is intended to stabilize its solution. This is achieved by constructing the following functional:

$$
f(s)=\|R s-m\|^{2}+k^{2}\|L s\|^{2} .
$$

The idea is to define the regularized solution $s$ as the minimizer of the following weighted combination of the residual norm and the side constraint:

$$
s=\arg \min \left\{\|R s-m\|_{2}^{2}+k\|L s\|_{2}^{2}\right\},
$$

where the regularization parameter, $k_{s}$ controls the weight given to the minimization of the side constraint relative to the minimization of the residual norm. $L$ parameter takes different forms in accordance with the order of regularization. In this paper, it has been chosen zero order Tikhonov regularization, $L=I$, the identity matrix.

The quality of the result of this method depends strongly on the regularization parameter. For this reason, a reliable method to determine this parameter is crucial to solve the inverse problem. One way to determine it is to plot the $L$-curve $e^{9-11)}$ as it can be seen in Fig. 3. In this curve, the 2-norm of the solution vector is plotted versus the 2-norm of the residual vector for different values of $k$ parameter. The optimal truncation parameter, $k$, is the closest to the maximum curvature point, that is, the value corresponding to the L-shaped corner, $k=0.001$ for our calculations. The corner value minimizes the two mentioned vectors at the same time.

A sensitivity analysis has been done to demonstrate that small changes in the value of $k$ affect the unfolded spectrum.

It has been calculated the deviation between the unfolded spectrum respect to the theoretical spectrum in terms of the Root Mean Squared (RMS), varying the $k$ parameter. Figure 4 shows the values of RMS obtained for different $k$ parameters. As it can be seen, the lower value of RMS is for the $k$ obtained with the $L$-curve criterion.

\section{Quality Parameters}

Unfolded spectrum can provide useful information that can be tested in routinary QC processes of X-ray tubes. Thus, HVL, homogeneity factor and mean energy have been studied. 


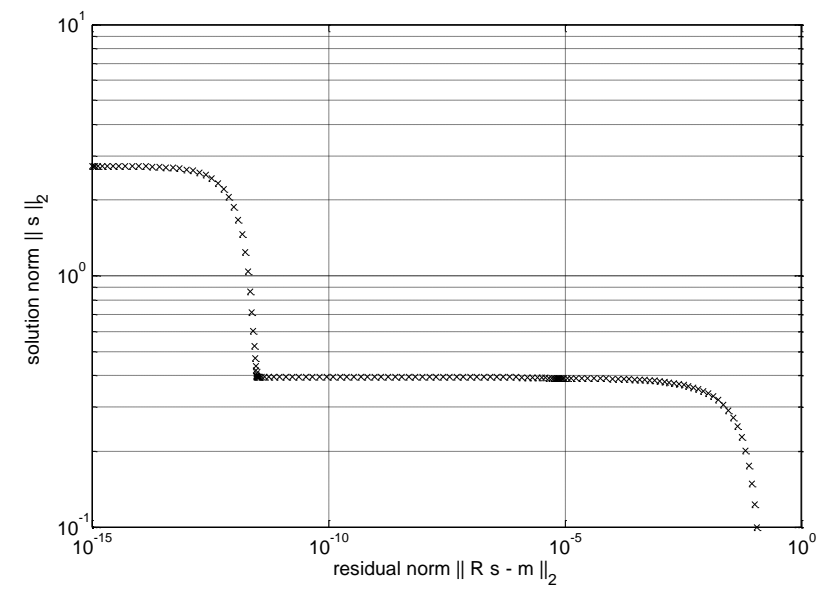

Fig. 3 L-curve shape

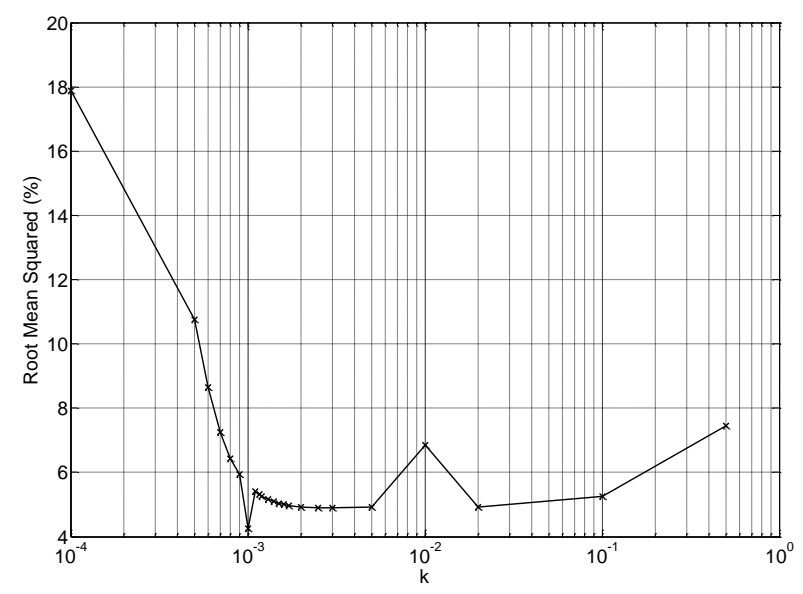

Fig. 4 Sensitivity analysis of $k$

The HVL is defined for different quantities: photon fluence, energy fluence, air kerma or absorbed dose.

Normally, the HVL is experimentally obtained by overlapping aluminum or copper foils of certain thickness and certified purity between the X-ray focus and an ionization chamber. However, HVL can also be determined by calculation if the primary X-ray spectrum is known. First HVL for air kerma $^{12,13)}$ is calculated for X-ray according to the following expression:

$$
1 / 2=\frac{\sum_{i}\left(\mu_{t r} / \rho\right)_{\text {air }} N_{i} h v_{i} \exp \left(-\mu_{A l} H V L\right) \exp \left(-\mu_{A i r} d\right)}{\sum_{i}\left(\mu_{t r} / \rho\right)_{\text {air }} N_{i} h v_{i} \exp \left(-\mu_{\text {Air }} d\right)},
$$

where

$\left(\mu_{t r} / \rho\right)_{a i r}$ is the mass energy-transfer coefficient in air. ${ }^{14)}$

$d \quad$ is the distance between the X-ray focus and the detector, normally an ionization chamber.

$h v_{i}$ is the photon energy of the $i$-th interval.

$N_{i}$ is the number of photons in the $i$-th energy interval. $\mu_{A l}$ is the linear attenuation coefficient in aluminum. ${ }^{14)}$

Reducing the air kerma to $1 / 4$, the second HVL can be obtained. Homogeneity factor is defined for each voltage as the ratio between the first and the second HVL. The mean photon energy is calculated from the expression:

$$
\overline{h v}=\frac{\sum_{i} N_{i} h v_{i}}{\sum_{i} N_{i}},
$$

where $h v_{i}$ is the photon energy of the $i$-th interval and $N_{i}$ is the number of photons in the $i$-th energy interval. For a given photon spectrum the mean photon energy is an important parameter because it represents the chromatic quality of the spectrum.

\section{Results and Discussion}

High voltage, filter thickness and anode angle have been analyzed to test the behavior of the unfolding method applied. Working conditions tested in each simulation are listed in Table 1.

Table 1 Working conditions tested

\begin{tabular}{cccc}
\hline Case & $\begin{array}{c}\text { Voltage } \\
(\mathrm{kVp})\end{array}$ & $\begin{array}{c}\text { Filter } \\
\text { thickness } \\
(\mathrm{mm} \mathrm{Al})\end{array}$ & $\begin{array}{c}\text { Anode } \\
\text { angle }\end{array}$ \\
\hline Case 1 & 78 & 5.0 & $12^{\circ}$ \\
Case 2 & 98 & 5.0 & $12^{\circ}$ \\
Case 3 & 150 & 5.0 & $12^{\circ}$ \\
Case 4 & 100 & 2.5 & $12^{\circ}$ \\
Case 5 & 100 & 4.5 & $12^{\circ}$ \\
Case 6 & 90 & 5.0 & $8^{\circ}$ \\
Case 7 & 90 & 5.0 & $12^{\circ}$ \\
\hline
\end{tabular}

\section{High Voltage Analysis}

Unfolded spectra obtained with the Tikhonov method are represented in Figs. 5, 6 and 7 respectively for 78, 98 and $150 \mathrm{kVp}$ together with theoretical spectra. For these spectra, the anode angle is $12^{\circ}$ and the anode material is Tungsten with $5 \mathrm{~mm}$ Aluminum filter.

Comparison shows slight differences that can be attributed to small mathematical errors in the Monte Carlo simulation and errors associated to the truncated pseudo-inverse. Nevertheless, tungsten characteristic peaks and the continuous Bremsstrahlung are well predicted.

Regarding to the characteristic lines, $\mathrm{K}_{\alpha 2}(57.9 \mathrm{keV})$ is well fitted in resolution (FWHM) and height. However, in the $\mathrm{K}_{\alpha 1}$ $(59.3 \mathrm{keV})$ it is observed a tail in the higher energy side of the peak that distorts it from the original Gaussian-shape. These discrepancies can be attributed to the width of the energy bin used to obtain the Response matrix. A wider energy bin implies smaller and wider peaks. If the energy bin was reduced lower discrepancies would be expected.

It is important to remark that the strong variation produced in the spectrum in the region of interest defined by characteristic lines, increases the ill condition of the Response matrix.

Regarding to L characteristic lines, they are not present in the considered spectra due to the inherent and added filter of the X-ray tube.

As it can be seen in Figs. 5, 6 and 7 the underestimation of $\mathrm{K}_{\beta}$ characteristic lines is more evident when the voltage is increased and it affects to the Gaussian-shape of $\mathrm{K}_{\alpha 1}$, especially when the voltage is higher than $80 \mathrm{kVp}$. In Fig. 7 it can 


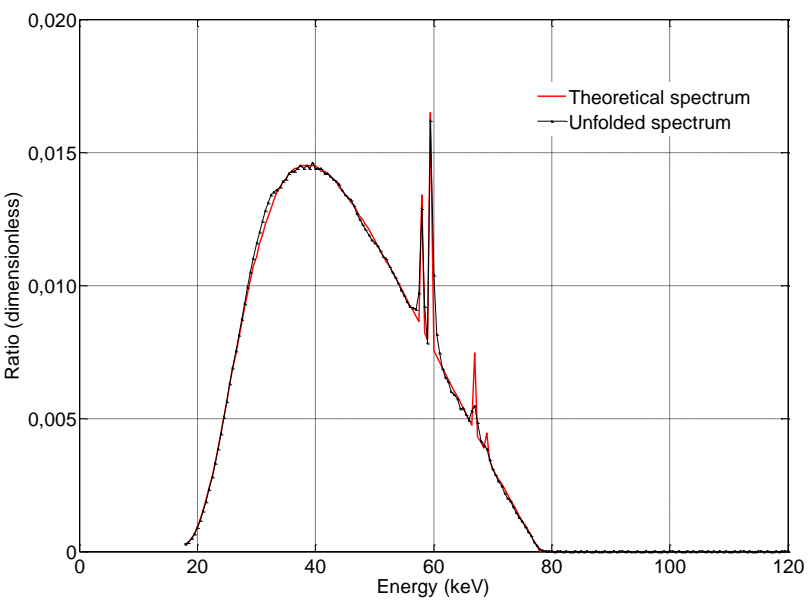

Fig. 5 Theoretical and unfolded spectrum for Case 1

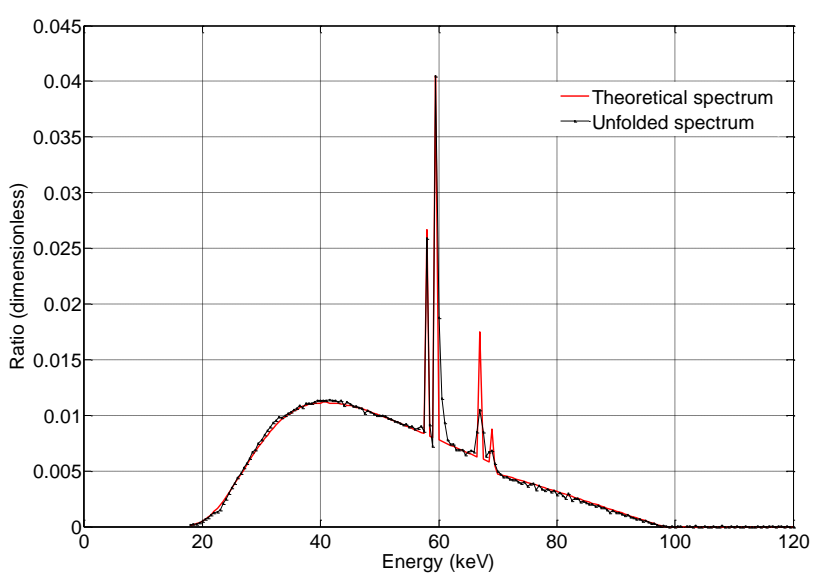

Fig. 6 Theoretical and unfolded spectrum for Case 2

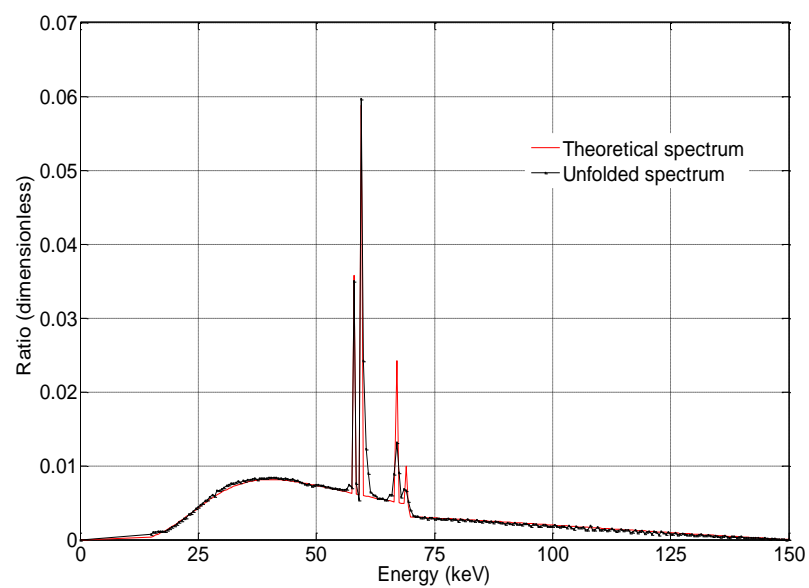

Fig. 7 Theoretical and unfolded spectrum for Case 3

be seen that for $150 \mathrm{kVp}$ this effect is more pronounced.

The effect of these discrepancies is analyzed from the point of view of spectrum quality parameters in Section III. 4.

When an unfolding method is used it is important to know the resolution achievable in the unfolded spectrum. For this purpose, two unfolded spectra have been compared only varying the voltage $2 \mathrm{kV}$. For small voltages variations, the spectrum mainly changes in the high energy region. In Fig. 8

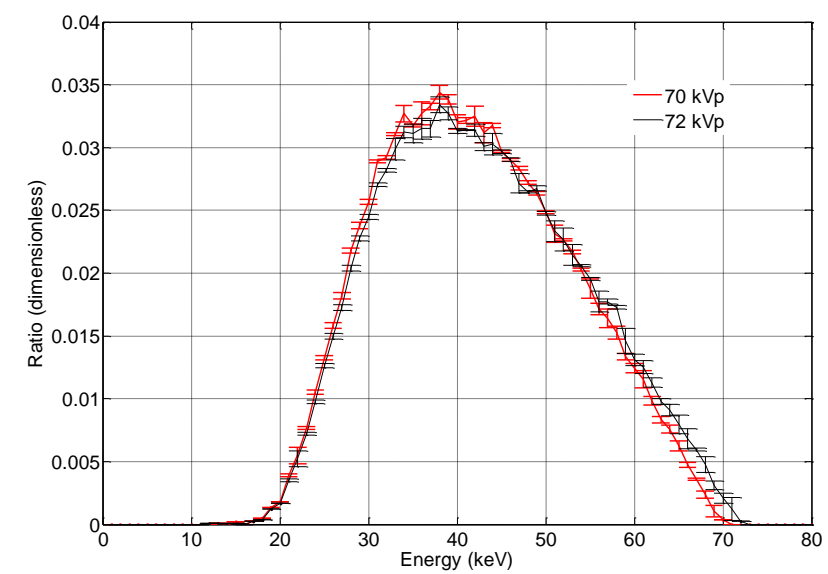

Fig. 8 Comparison of two unfolded spectra produced by 70 and $72 \mathrm{kVp}$

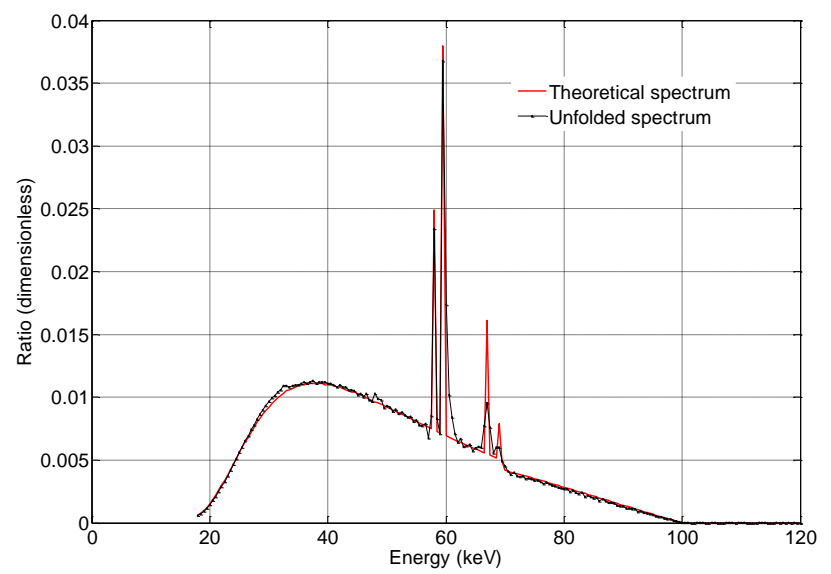

Fig. 9 Theoretical and unfolded spectrum for Case 3

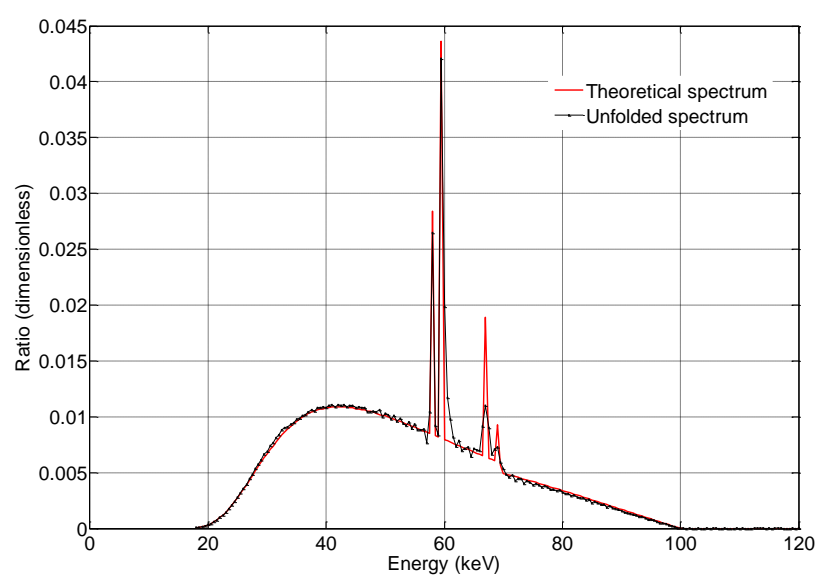

Fig. 10 Theoretical and unfolded spectrum for Case 4

it is shown a comparison of two unfolded spectra corresponding to 70 and $72 \mathrm{kVp}$. $2 \sigma$ error bars have been added to the graph to find the regions where both spectra overlap.

For smaller variations in voltage, $2 \sigma$ error bars overlaps in the entire energy range. Therefore, it is needed at least $2 \mathrm{kV}$ in order to distinguish two unfolded spectra at least in the high energy region. 


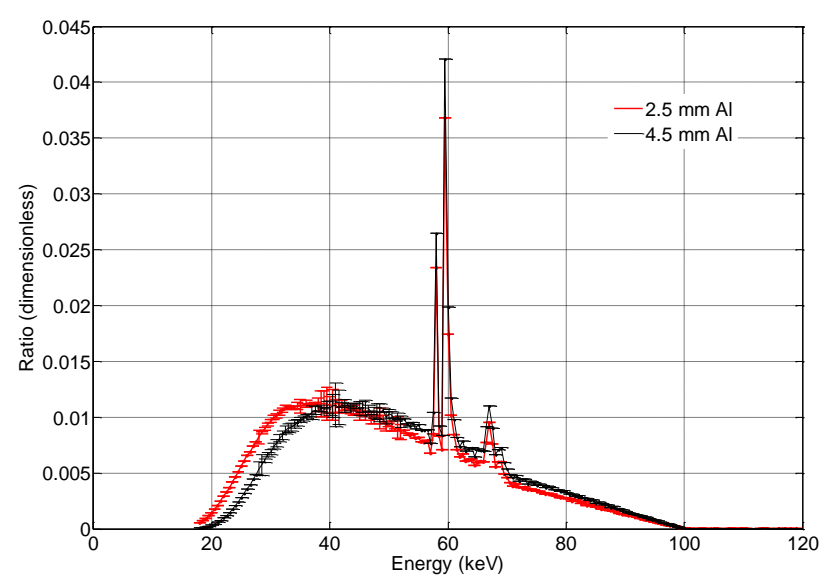

Fig. 11 Comparison of two unfolded spectra produced by 2.5 and $4.5 \mathrm{~mm}$ Aluminum thickness: $11^{\circ}$ anode angle

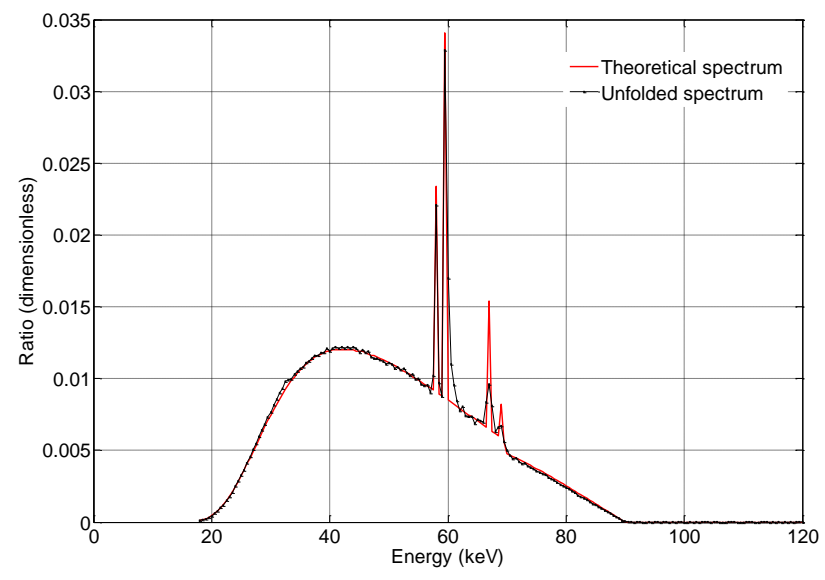

Fig. 12 Theoretical and unfolded spectrum for Case 5

\section{Filter Thickness Analysis}

Two different thickness of Aluminium filter have been tested for $100 \mathrm{kVp}$ and $12^{\circ}$ anode angle. Results of comparison between IPEM 78 and unfolded spectra are shown in Figs. 9 and 10, respectively for 2.5 and $4.5 \mathrm{~mm}$.

The effect of increasing the filter thickness produces a shifting of the spectrum to higher energies, as it can be seen comparing Figs. 9 and 10. No special behaviour of the Tikhonov method has been noted analysing the unfolded spectra when the filter thickness is changed.

Comparison of two unfolded spectra obtained for 2.5 and $4.5 \mathrm{~mm} \mathrm{Al}$ with $2 \sigma$ error bars are presented in Fig. 11 to study again the resolution of the unfolding method. It can be stated that a minimum change of $2 \mathrm{~mm}$ in thickness is required in order to avoid the overlapping of $2 \sigma$ error bars.

\section{Anode Angle Analysis}

The third parameter studied is the variation in the anode angle. Two unfolded spectra have been obtained for $8^{\circ}$ and $12^{\circ}$ anode angle. In both cases $90 \mathrm{kVp}$ and $5 \mathrm{~mm}$ Aluminium filter have been considered. Unfolded spectra compared with theoretical ones are presented in Figs. 12 and 13, respectively for the selected anode angles

The effect of varying the anode angle apparently does not

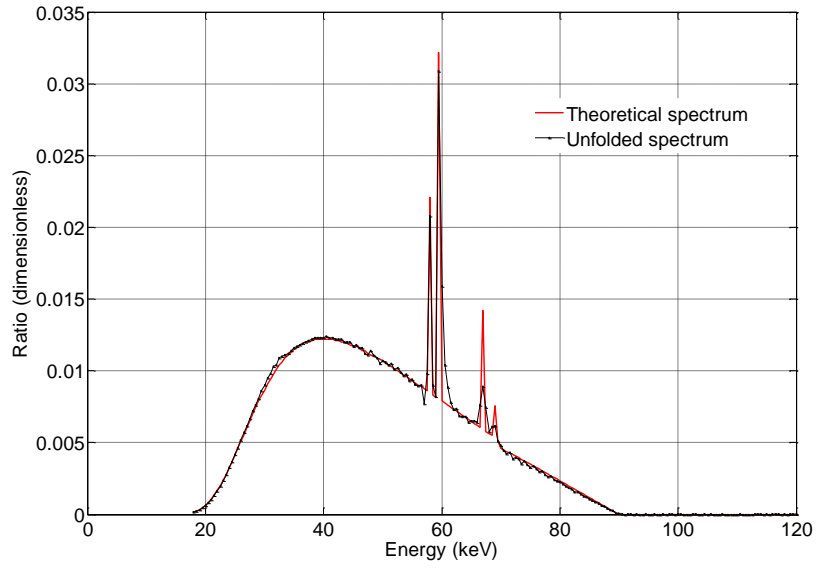

Fig. 13 Theoretical and unfolded spectrum for Case 6

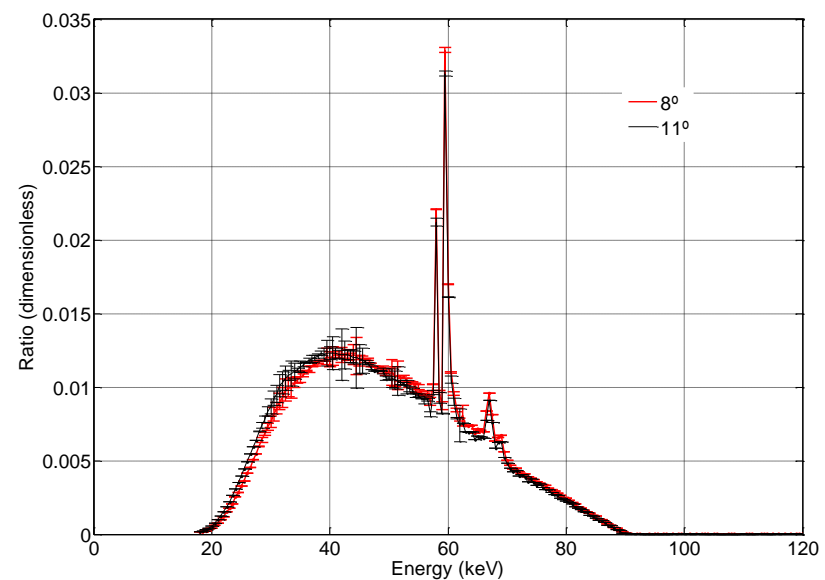

Fig. 14 Comparison of two unfolded spectra produced by 8 and $11^{\circ}$ anode angle

change the behaviour of the Tikhonov method. Anyway, this effect is less significant in the spectrum shape and it is only observed in the low energy region. The resolution associated with Tikhonov method when the anode angle is changed has been analyzed by varying the angle in one degree steps. It is necessary a $3^{\circ}$ variation to avoid overlapping of two unfolded spectra with $2 \sigma$ error bars as it can be seen in Fig. 14.

\section{Quality Parameter Analysis}

To have a quantitative understanding of the deviation of the unfolded spectra from the theoretical IPEM spectrum, the Root Mean Squared (RMS), have been applied to estimate the good quality of fitting. This method is used to evaluate whether or not the observed variations in the spectrum of $n$

Table 2 Root Mean Squared (\%)

\begin{tabular}{cc}
\hline Case & RMS \\
\hline Case 1 & 4.26 \\
Case 2 & 3.03 \\
Case 3 & 3.76 \\
Case 4 & 3.01 \\
Case 5 & 3.04 \\
Case 6 & 3.17 \\
Case 7 & 3.50 \\
\hline
\end{tabular}


Table 3 Quality parameters for high voltage variation

\begin{tabular}{cccc}
\hline $\begin{array}{c}\text { High } \\
\text { Voltage }\end{array}$ & $\begin{array}{c}\text { Quality pa- } \\
\text { rameters }\end{array}$ & $\begin{array}{c}\text { IPEM78 } \\
\text { spectrum }\end{array}$ & $\begin{array}{c}\text { TIKHONOV un- } \\
\text { folded spectrum }\end{array}$ \\
\hline \multirow{7}{*}{$78 \mathrm{kVp}$} & First HVL & 3.400 & $3.180(6.47)$ \\
& Second HVL & 8.030 & $7.780(3.11)$ \\
& Hom factor & 0.423 & $0.408(3.55)$ \\
& Mean Energy & 45.01 & $44.46(1.22)$ \\
& First HVL & 4.350 & $4.090(5.98)$ \\
$98 \mathrm{kVp}$ & Second HVL & 10.450 & $10.120(3.16)$ \\
& Hom factor & 0.416 & $0.404(2.88)$ \\
& Mean Energy & 51.74 & $52.54(-1.55)$ \\
& First HVL & 4.150 & $4.010(3.37)$ \\
$150 \mathrm{kVp}$ & Second HVL & 11.28 & $10.91(3.28)$ \\
& Hom factor & 0.368 & $0.356(3.26)$ \\
& Mean Energy & 59.47 & $60.15(-1.14)$ \\
\hline
\end{tabular}

Table 4 Quality parameters for anode angle variation

\begin{tabular}{cccc}
\hline $\begin{array}{c}\text { Anode } \\
\text { angle }\end{array}$ & $\begin{array}{c}\text { Quality parame- } \\
\text { ters }\end{array}$ & $\begin{array}{c}\text { IPEM78 } \\
\text { spectrum }\end{array}$ & $\begin{array}{c}\text { TIKHONOV } \\
\text { unfolded spectrum }\end{array}$ \\
\hline \multirow{4}{*}{$8^{\circ}$} & First HVL & 4.320 & $4.020(6.94)$ \\
& Second HVL & 10.260 & $9.840(4.09)$ \\
& Hom factor & 0.421 & $0.409(2.85)$ \\
& Mean Energy & 50.36 & $50.91(-1.09)$ \\
$12^{\circ}$ & First HVL & 4.120 & $3.880(5.83)$ \\
& Second HVL & 9.820 & $9.480(3.46)$ \\
& Hom factor & 0.420 & $0.409(2.62)$ \\
& Mean Energy & 49.72 & $50.28(-1.13)$ \\
\hline
\end{tabular}

Table 5 Quality parameters for filter thickness variation

\begin{tabular}{cccc}
\hline $\begin{array}{c}\text { Filter } \\
\text { thickness }\end{array}$ & $\begin{array}{c}\text { Quality para- } \\
\text { meters }\end{array}$ & $\begin{array}{c}\text { IPEM78 } \\
\text { spectrum }\end{array}$ & $\begin{array}{c}\text { TIKHONOV un- } \\
\text { folded spectrum }\end{array}$ \\
\hline & First HVL & 3.560 & $3.430(3.65)$ \\
$2.5 \mathrm{~mm} \mathrm{Al}$ & Second HVL & 8.960 & $8.770(2.12)$ \\
& Hom factor & 0.397 & $0.391(1.51)$ \\
& Mean Energy & 49.97 & $50.69(-1.44)$ \\
& First HVL & 4.69 & $4.42(5.76)$ \\
$4.5 \mathrm{~mm} \mathrm{Al}$ & Second HVL & 11.16 & $10.79(3.32)$ \\
& Hom factor & 0.420 & $0.410(2.38)$ \\
& Mean Energy & 52.99 & $53.76(-1.45)$ \\
\hline
\end{tabular}

data points are within an acceptable range. Results of RMS in $\%$ are listed in Table $\mathbf{2}$ where we can see that all values are lower than $4.5 \%$.

On the other hand, quality parameters such as first and second HVL, homogeneity factor and mean energy have been calculated for both theoretical and unfolded spectra. Results are listed in Tables 3, 4 and 5, respectively, for high voltage, filter thickness and anode angle variation. Relative errors between Tikhonov and IPEM78 spectra appear into brackets in $\%$.

Discrepancies in First and Second HVL are lower than $7 \%$. These discrepancies are attributed to the disagreement in the estimation of $\mathrm{K}_{\beta}$ characteristic lines. An analogous conclusion can be achieved in the case of the homogeneity factor. On the other hand, the relative error calculated for the mean energy spectrum is less than $2 \%$ for all cases.
From these results, it can be stated that the error associated to the Tikhonov unfolding method produce a variation in quality parameters less than $7 \%$.

To estimate the effect of these discrepancies, another MCNP model has been developed to calculate the dose in a water voxel $\left(1 \times 1 \times 1 \mathrm{~cm}^{3}\right)$ at $30 \mathrm{~cm}$ from the X-ray focus. $\mathrm{F} 4$ Tally has been obtained in the water voxel and converted into dose using conversion factors of photon fluence to dose ${ }^{14)}$. The relative difference respect to the dose calculated with the theoretical spectrum is less than $5 \%$ in any case.

\section{Conclusion}

A sensitivity analysis has been performed to know the influence of the Tikhonov method on the reconstructed spectrum as well as on quality parameters.

When the high voltage is increased the unfolded spectrum shows lower peaks for $\mathrm{K}_{\beta}$ characteristic lines as well as a small distortion on $\mathrm{K}_{\alpha}$ characteristic lines.

The Tikhonov method seems to be not sensitive to changes in filter thickness and anode angle.

A comparison between original and reconstructed spectra shows values of RMS lower than $4.5 \%$. These differences have been evaluated in terms of dose to patient being the influence lower than 5\%.

An analysis of quality parameters gives similar errors for both HVLs while they are lower for homogeneity factor and mean energy. These values are lower than those obtained with Modified Truncated Singular Value Decomposition studied in previous papers.

Therefore, the Tikhonov unfolding method reproduces properly primary spectrum and associated quality parameters.

\section{Acknowledgment}

This work has been partially supported by the Valencian Region Government under project Grant GVPRE/2008/136, and the Universidad Politécnica de Valencia under project PAID-06-07-3300.

\section{References}

1) G. Matscheko, R. Ribberfors, "A Compton scattering spectrometer for determining x-ray photon energy spectra," Phys. Med. Biol., 32[5], 577-594 (1987).

2) S. Gallardo, J. Ródenas, G. Verdú, "Monte Carlo simulation of the Compton scattering technique applied to characterise diagnostic X-ray spectra," Med. Phys., 31[7], 2082-2090 (2004).

3) X-5 MONTE CARLO TEAM, MCNP - A General Monte Carlo Nparticle Transport Code, Version 5 LA-UR-03-1987, Los Alamos National Laboratory (LANL) (2003).

4) S. Gallardo, D. Ginestar, G. Verdú, J. Ródenas, V. Puchades and J. I. Villaescusa, "X-ray spectrum unfolding using a regularized truncated SVD method," X-ray Spectrom., 35, 63-70 (2006).

5) IPEM Report 78, Catalogue of Diagnostic X-Ray Spectra \& Other Data, Institute of Physics and Engineering in Medicine. (CD-Rom Edition 1997).

6) SPECTRO-X -X-ray Spectrum Analyser. Compton Spectrometer Manual. RTI Electronics. Sweden (1994). 
7) Canberra, http://www.canberra.com

8) G. H. Golub, C.F. Van Loan, Matrix Computations, The Johns Hopkins University Press, Third edition, (1996).

9) P. C. Hansen, "Regularization tools," Version 3.0 for Matlab 5.2, Numer. Algorithms, 20, 195-196 (1999).

10) P. C. Hansen, "A Matlab package for analysis and solution of discrete ill-posed problems," Numer. Algorithms, 6[1], 1-35 (1994).

11) P. C. Hansen, Rank-Deficient and Discrete Ill-Posed Problems, Society for Industrial and Applied Mathematics (SIAM),
Philadelpia (1998).

12) W. Abdel-Rahman, E. B. Podgorsak, "Energy transfer and energy absorption in photon interactions with matter revisited: A step-by-step illustrated approach," Radiat. Phys. Chem., 79[5], 552-566 (2010).

13) E. Mainegra-Hing, I. Kawrakow, "Efficient x-ray tube simulations," Med. Phys., 33[8], 2683-2690 (2006).

14) X-Ray Attenuation and Absorption for Materials of Dosimetric Interest, National Institute of Standards and Technology (NIST). (Last updated 2010). 